\title{
MAGNETISATION OF Co/Ti MULTILAYERS
}

\author{
K. SMARDZ ${ }^{a}$, L. SMARDZ ${ }^{b}$ AND A. JEZIERSKI ${ }^{b}$ \\ ${ }^{a}$ Inst. of Mater. Sci. and Eng., University of Technology \\ Pl. Curie 5, 60-965 Poznań, Poland \\ ${ }^{b}$ Institute of Molecular Physics, Polish Academy of Sciences \\ Smoluchowskiego 17, 60-179 Poznań, Poland
}

We studied experimentally and theoretically the magnetisation of the $\mathrm{Co} / \mathrm{Ti}$ multilayers. The results showed that the magnetic moments on Co atoms located at the Co-Ti interface are strongly reduced. For $d_{T_{i}}=0.35 \mathrm{~nm}$ the estimated effective non-magnetic Co thickness at the Co-Ti interface amounts $\approx 0.08 \mathrm{~nm}$ and $\approx 0.1 \mathrm{~nm}$ at $4 \mathrm{~K}$ and $295 \mathrm{~K}$, respectively. We found a good agreement between experimentally determined and theoretically calculated magnetisations.

PACS numbers: 75.70.-i, 75.30.Et

Metallic multilayers (MLs) composed of alternating sublayers of ferromagnetic and non-magnetic metals have been the subjects of great interest in the last few years. This is mainly due to their interesting magnetic properties, for example: giant magnetoresistance, oscillating interlayer exchange coupling, and perpendicular anisotropy. These properties can be tailored by varying the composition and thickness of the magnetic and non-magnetic sublayers. For potential application, the coercivity and magnetisation of multilayered structures is an important parameter. For instance, such a structure for magnetic head application should be magnetically soft exhibiting high saturation magnetisation [1]. Recently we have showed $[2,3]$ that below the critical Co thickness $d$, the Co/ $\operatorname{Zr}(d \approx 2.8 \mathrm{~nm})$ and Co/Ti $(d \approx 3 \mathrm{~nm})$ MLs are magnetically soft and exhibit a saturation magnetisation higher than that observed in conventional soft magnetic films. It has been found that up to the critical thickness, Co sublayers grow in the soft magnetic nanocrystalline structure, and above in the polycrystalline structure with the average Co grain size greater than the magnetic exchange length $(L \approx 10 \mathrm{~nm})[2,3]$. In this paper we report on the magnetisation studies of the Co/Ti MLs using the experimentally determined total magnetic moment of the samples and theoretical calculations of the magnetic moment distribution on the Co and $\mathrm{Ti}$ atoms.

The Co/Ti MLs with constant-thicknesses sublayers were deposited onto $\mathrm{Si}(111)$ and glass substrate at room temperature (RT) using an UHV $\left(5 \times 10^{-10} \mathrm{mbar}\right)$ magnetron sputtering. The Co-layers $\left(0.2 \leq d_{c_{0}} \leq 20 \mathrm{~nm}\right)$ were deposited using a dc source. For preparation of Ti-layẹr $\left(0<d_{\mathrm{Ti}}<10 \mathrm{~nm}\right)$ an $\mathrm{RF}$ source was used. The number of repetitions was increased with decreasing Co thickness, so as to keep the total Co thickness equal to about $100 \mathrm{~nm}$. The chemical 
composition and the cleanness of all layers was checked in situ, immediately after deposition, transferring the MLs to an UHV $\left(4 \times 10^{-11} \mathrm{mbar}\right)$ analyse chamber equipped with Auger and X-ray photoelectron spectroscopy (AES, XPS). From the exponential variation of the XPS Co- $2 p$ and Ti- $2 p$ integral intensities with increasing layer thickness we conclude that the Co and Ti sublayers grow homogeneously. The structure of the samples was examined $e x$ situ by standard $\theta-2 \theta \mathrm{X}$-ray diffraction with Co $K_{\alpha}$ radiation. The modulation wavelength was determined from the spacing between satellite peaks in the low-angle X-ray diffraction patterns. The results were consistent with values obtained from total thickness divided by the number of repetitions. The thicknesses of individual Co and $\mathrm{Ti}$ sublayers were also determined using $\mathrm{X}$-ray fluorescence analysis. The total magnetic moment of the $\mathrm{Co} / \mathrm{Ti}$ MLs was measured using a vibrating sample magnetometer in the temperature range $4-300 \mathrm{~K}$. The local magnetic moments on $\mathrm{Co}$ and $\mathrm{Ti}$ atoms have been calculated by the spin-polarised self-consistent tight binding linear muffin tin orbital method [4].

For the Co/Ti MLs with $d_{\mathrm{Co}}>3 \mathrm{~nm}$ the high-angle X-ray diffraction patterns show (002) reflections of hcp Co and Ti. The average Co and Ti crystallite sizes in direction perpendicular to the substrates, as determined from the Scherrer equation, are comparable to their respective sublayer thicknesses. Only a broad peak related to the Ti sublayers was observed for the MLs with $d_{\mathrm{Co}}<3 \mathrm{~nm}$ and $d_{\text {Ti }}>2 \mathrm{~nm}$, in agreement with X-ray diffraction studies reported in Ref. [3]. For $d_{\mathrm{Co}}<3 \mathrm{~nm}$ and $d_{\mathrm{Ti}}<2 \mathrm{~nm}$, practically no reflections was observed in the high-angle X-ray diffraction patterns. These results confirm that for $d_{\mathrm{Co}}<$ $3 \mathrm{~nm}$ the Co sublayers grow in the nanocrystalline phase with average grains size $D \ll 10 \mathrm{~nm}$ [3]. We have previously observed very similar growth mode of the Co sublayers for the Co/Zr MLs [2].

Figure 1a shows the magnetisation of Co/Ti MLs with $d_{\mathrm{Ti}}=0.35 \mathrm{~nm}$, measured at $295 \mathrm{~K}$ (circles) and $4 \mathrm{~K}$ (squares), as a function of the cobalt sublayer thickness. We have determined the experimental magnetisation of the Co sublayers relative to the magnetisation of the pure $100 \mathrm{~nm}$ Co film with exactly the same area as the studied Co/Ti MLs $(5 \mathrm{~mm} \times 10 \mathrm{~mm})$. The reference $100 \mathrm{~nm}$ Co film showed magnetisation equal to the bulk hcp Co value. The magnetisation of the MLs slightly decreases with the decrease in the Co sublayer thickness down to about $3 \mathrm{~nm}$. For smaller Co thickness magnetisation decreases very rapidly. The above effect could be explained by the reduced magnetic moments on the Co atoms located near the $\mathrm{Ti}$ sublayers or (and) formation of a non-magnetic alloy at the Co-Ti interface.

The theoretical calculations of the local magnetic moments on Co and $\mathrm{Ti}$ atoms in the Co/Ti MLs were performed for the Ti sublayer thickness fixed to 1, 2 , or 3 atomic layers (AL). Furthermore, we assumed a rectangular modulation profile ("sharp" Co-Ti interface) of the Co/Ti MLs. The results showed that the local magnetic moments on Co atoms located at the Co-Ti interface are strongly reduced. The calculated values of the moments on the interface Co atoms amount $0.99,0.83$, and $0.81 \mu_{\mathrm{B}}$ for Ti sublayer thicknesses equal to 1,2 , and $3 \mathrm{AL}$, respectively. The magnetic moment on Co atoms, located in the third and more remote layers from the $\mathrm{Co}-\mathrm{Ti}$ interface, was practically equal to the bulk value. We also 


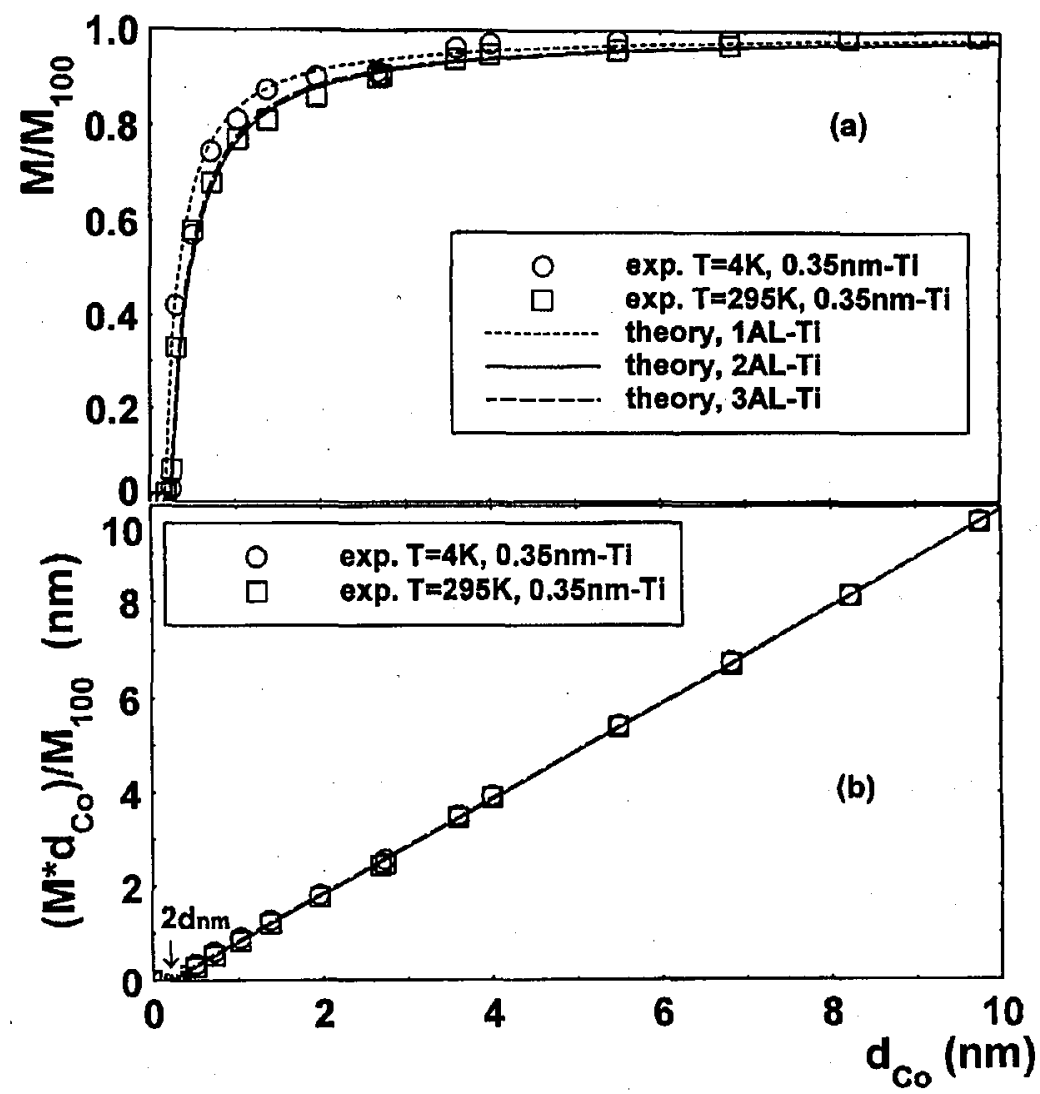

Fig. 1. Magnetisation (a) and plot $\left(M^{*} d_{\mathrm{Co}}\right) / M_{100}$ (b) versus Co layer thickness (see text) for the $d_{\mathrm{Co}}-\mathrm{Co} / 0.35 \mathrm{~nm}-\mathrm{Ti}$ multilayers.

observed a small induced magnetic moment (antiparallel to the Co moment) on $\mathrm{Ti}$ atoms located near the Co sublayer.

The theoretical thickness dependence of the magnetisation, calculated relative to the magnetisation of the $100 \mathrm{~nm}$ Co film, is shown in Fig. 1a (solid or broken lines). As the average Ti sublayer thickness $(0.35 \mathrm{~nm}$ ) corresponds to 1 or $2 \mathrm{AL}$, the agreement between experimental (circles) and theoretical magnetisations (see Fig. 1a) is good. The experimental magnetisation of the Co/Ti MLs can be described by a simple model with a non-magnetic ("dead") ultrathin layer at the Co-Ti interface. In this model the Co sublayer of thickness $d_{\text {Co }}$ is divided into a non-magnetic layer of thickness $d_{\text {nm }}$ at each Ti interface and ferromagnetic layer of thickness $d_{\mathrm{Co}}-2 d_{\mathrm{nm}}$ at the centre of the Co layer. The magnetisation of the Co sublayers $(M)$ relative to the magnetisation of pure $100 \mathrm{~nm}$ Co film $\left(M_{100}\right)$ can be written as follows:

$$
M / M_{100}=\left(d_{\mathrm{Co}}-2 d_{\mathrm{nm}}\right) / d_{\mathrm{Co}} .
$$

To estimate the effective non-magnetic Co layer thickness at the Co-Ti interface $\left(d_{\mathrm{nm}}\right)$, we used Eq. (1) in the form: $M^{*} d_{\mathrm{Co}} / M_{100}=d_{\mathrm{Co}}-2 d_{\mathrm{nm}}$. 
Figure 1b shows a plot of $M^{*} d_{\mathrm{Co}} / M_{100}$ versus Co sublayer thickness $d_{\text {Co. }}$. From the linear regression fit to the data, which show $M>0$, we estimated an effective non-magnetic Co layer thickness at the Co-Ti interface, as $d_{\mathrm{nm}} \approx 0.08 \mathrm{~nm}$ (broken line) and $0.1 \mathrm{~nm}$ (solid line) at $4 \mathrm{~K}$ and $295 \mathrm{~K}$, respectively. The thickness of the effective non-magnetic Co layer, determined in our experiment, is smaller than that reported in Ref. [5] $\left(0.55 \mathrm{~nm}\right.$ for $\left.d_{\mathrm{Ti}}=2.1 \mathrm{~nm}\right)$ and much smaller compared to that reported in Ref. [6] $\left(1.13 \mathrm{~nm}\right.$ for $\left.d_{\mathrm{Ti}}=4.9 \mathrm{~nm}\right)$. The smaller effective non-magnetic Co layer thickness could be associated with thinner $\mathrm{Ti}$ sublayer or low energy of atoms at the substrate. The latter condition was satisfied for our sputtering methods due to a large target-substrate distance $(\approx 220 \mathrm{~mm})$.

As we have already mentioned above, the alloying effect during the growth process could be also responsible for the existence of the effective non-magnetic Co layer at the Co-Ti interface. Such an effect was revealed very recently for the "as deposited" Co/Ti MLs, but only for the thicker Ti sublayers $\left(d_{\mathrm{Ti}}=2.5 \mathrm{~nm}\right)[7,8]$. On the other hand, the difference between the estimated $d_{\mathrm{nm}}$ values at $4 \mathrm{~K}$ and $295 \mathrm{~K}$ is very small and lies in the experimental error limit. We can accordingly neglect the alloying effect for the Co/Ti MLs with $d_{T i}=0.35 \mathrm{~nm}$. Therefore, we conclude that the thickness dependence of the experimental magnetisation shown in Fig. 1a could be explained by a strong reduction of the magnetic moments on the interface Co atoms, in accordance with the theoretical results.

In summary, we have estimated an effective non-magnetic Co thickness at the Co-Ti interface as $\approx 0.08 \mathrm{~nm}$ and $\approx 0.1 \mathrm{~nm}$ at $4 \mathrm{~K}$ and $295 \mathrm{~K}$, respectively. The results showed that the magnetic moments on Co atoms located at the $\mathrm{Co}-\mathrm{Ti}$ interface are strongly reduced. The agreement between experimentally determined and theoretically calculated magnetisations of Co/Ti MLs with $d_{\mathrm{Ti}}=0.35 \mathrm{~nm}$ is good.

\section{Acknowledgments}

This work was in part financially supported by the Committee for Scientific Research under grant No. 7 T08C 00914.

\section{References}

[1] M. Hayakawa, J. Magn. Magn. Mater. 134, 287 (1994).

[2] L. Smardz, K. Le Dang, H. Niedoba, K. Chrzumnicka, J. Magn. Magn. Mater. 140-144, 569 (1995).

[3] K. Smardz, Ph.D. thesis, Institute of Molecular Physics, PAS, Poznan 1999.

[4] O.K. Andersen, O. Jepsen, Phys. Rev. Lett. 53, 2571 (1984).

[5] P. Wu, E.Y. Jiang, H.L. Bai, H.Y. Wang, C.D. Wang, Phys. Status Solidi A 161, 125 (1997).

[6] R. Van Leeuwen, C.D. England, J.R. Dutcher, C.M. Falco, W.R. Bennett, B. Hillebrands, J. Appl. Phys. 67, 4910 (1990).

[7] G.M. Lee, K.W. Kim, Y.V. Kudryavtsev, L. Smardz, Y.P. Lee, Thin Solid Films 341, 165 (1999).

[8] Y.P. Lee, G.M. Lee, K.W. Kim, Y.V. Kudryavtsev, L. Smardz, J. Magn. Soc. Japan 23, 361 (1999). 